\title{
Preface: Queueing theory and network applications II
}

\author{
Tuan Phung-Duc ${ }^{1} \cdot$ Yutaka Takahashi ${ }^{2} \cdot$ Hideaki Takagi $^{1}$ \\ Published online: 24 April 2020 \\ (c) Springer Science+Business Media, LLC, part of Springer Nature 2020
}

This special issue of Annals of Operations Research is devoted to recent developments in queueing theory and network applications. We invited the authors of the papers presented at the 12th International Conference on Queueing Theory and Network Applications (QTNA2018), Tsukuba, Japan, July 25-27, 2018, to submit their extended versions. We also welcomed researchers who did not participate in the conference to submit their new contributions. As a result, 27 submissions from countries all over the world were submitted. After a rigorous review process, 16 papers were selected for inclusion in this special issue.

The special issue can be roughly divided into two groups: (1) recent advances in queueing theory and related stochastic models in the first eight papers and (2) novel stochastic analysis of network applications in the next eight papers.

We would like to thank all the authors who submitted their papers to this special issue. Furthermore, we also thank the referees who spent their time writing high quality reviews. Finally, we would like to thank Editor-in-Chief, Professor Endre Boros, for accepting our proposal to edit this special issue. We also sincerely thank the former Publications Manager Katie D'Agosta and the current Publications Manager Ann Pulido for their support and assistance.

\section{Guest Editors}

Tuan Phung-Duc

Yutaka Takahashi

Hideaki Takagi

Publisher's Note Springer Nature remains neutral with regard to jurisdictional claims in published maps and institutional affiliations.

Tuan Phung-Duc

tuan@ sk.tsukuba.ac.jp

Yutaka Takahashi

takahashi@i.kyoto-u.ac.jp

Hideaki Takagi

takagi@sk.tsukuba.ac.jp

1 University of Tsukuba, Tsukuba, Ibaraki, Japan

2 Kyoto University, Kyoto, Japan 